\title{
PRIVACIDADE E CONFIDENCIALIDADE NA ASSISTÊNCIA À SAÚDE DO ADOLESCENTE: PERCEPÇÖES E COMPORTAMENTOS DE UM GRUPO DE 711 UNIVERSTTÁRIOS
}

Jussara de Azambuja loch*, Joaquim Clotet, José Roberto Goldim

Trabalho realizado no Programa de Pós-Graduação em Ciências Médicas: Pediatria. Faculdade de Medicina/UFRGS.

*Correspondência

Rua Ramiro Barcelos, 2400

$2 \circ$ andar

Cep: 90035-003 -

Porto Alegre - RS

Tel: (51) 3316.5601

ppgped@vortex.ufrgs.br

\begin{abstract}
RESUMO
OBjetIvo. Conhecer as opiniões e comportamentos de um grupo de universitários sobre o grau de privacidade que consideram adequado em várias situações clínicas e em quais destas situações admitem a quebra de confidencialidade.

Métodos. Trata-se de um estudo transversal, descritivo, quali-quantitativo, no qual 711 universitários responderam a um questionário anônimo sobre a confidencialidade na assistência à saúde. O fator em estudo foi o reconhecimento dos limites éticos à confidencialidade e o desfecho avaliado foi a admissão da quebra de confidencialidade em situações clínicas freqüentes na morbidade dos adolescentes. A análise estatística utilizou o Epilnfo 6.04 e Microsoft Excel, 1997. A pesquisa foi aprovada pelo CEP/PUCRS

RESULTADOS. $82 \%$ dos adolescentes identificam a situação ideal de revelação mediante autorização do paciente, diferenciandoa das demais formas de quebra de confidencialidade. Em relação a revelações não autorizadas, a maioria admite a quebra do sigilo nas situações de idéias suicidas (85\%), violência (84,2\%), abuso sexual $(81,7 \%)$, anorexia nervosa $(81,3 \%)$ e risco à vida de terceiros (72,3\%); cerca da metade em HIV/Aids $(57,9 \%)$, drogadição $(51,7 \%)$ e DST $(44,7 \%)$; e menos de um terço a aceitam em casos de gravidez (33,6\%), homossexualidade $(20,7 \%)$ e atividade sexual $(15,6 \%)$.

Conclusäo. Os participantes estabelecem graus diferentes sobre 0 valor da confidencialidade no contexto assistencial. Aceitam que as informações sejam comunicadas a terceiros quando houver autorização do paciente. Quanto maiores os riscos à integridade física, mais facilmente admitem a quebra não autorizada do sigilo, porém dificilmente a aceitam nos aspectos referentes a sua sexualidade.
\end{abstract}

Unitermos: Adolescência. Privacidade. Comunicação sigilosa.

\section{INTRODUÇÃO}

A assistência ao adolescente é um desafio na prática médica contemporânea, tanto pelas características epidemiológicas de morbi-mortalidade desta faixa etária, como pelas controvérsias éticas, legais e sociais referentes aos direitos à privacidade e à confidencialidade da relação médico-adolescente.

Os graves problemas de saúde do grupo - que incluem doenças sexualmente transmissíveis, gravidez não desejada, uso ou abuso de drogas, depressão, suicídio, abuso sexual, violências e acidentes - estão, em grande parte, condicionados ao processo natural de desenvolvimento biopsicossocial da adolescência, o qual facilita a experimentação e a adoção de comportamentos de risco!. Nas últimas décadas, estes problemas têm aumentado em frequêencia e intensidade, atingindo adolescentes cada vez mais jovens. $\mathrm{Na}$ América do Norte, um em cada cinco adolescentes tem pelo menos um problema grave de saúde e um em cada quatro está em risco para o fracasso escolar, a delinqüência, o início precoce da vida sexual sem proteção adequada ou para o uso de drogas².

Várias pesquisas revelam que o principal motivo para o adolescente retardar a busca de auxílio especializado é sua preocupação com a confidencialidade e o receio de que o médico revele à família as informações sensíveis compartilhadas na consulta ${ }^{3-4}$.
A confidencialidade é tanto um direito do paciente quanto uma obrigação do médico. Pode ser definida como um tipo de privacidade informacional e está presente na assistência à saúde quando uma informação é revelada para o profissional no contexto da relação clínica e este, ao tomar ciência dela, compromete-se a não divulgá-la para terceiras partes sem a permissão do informante ${ }^{5}$. A confidencialidade não é prerrogativa de pacientes adultos. Aplica-se a todas as faixas etárias, como conseqüência de direitos éticos e legais à privacidade, os quais limitam o acesso de terceiros a um âmbito privado e íntimo da pessoa, seja através de contato físico ou da revelação de idéias, informações, fatos ou sentimentos ${ }^{5-7}$.

Em várias situações da assistência aos jovens, no entanto, os profissionais de saúde questionam o grau de sigilo que devem manter. Isto porque a capacidade para tomar decisões e fazer julgamentos adequados sobre questões de saúde dependem da presença de certas habilidades intelectivas e psicológicas que se estabelecem durante 0 processo de desenvolvimento cognitivomoral do indivíduo, só atingindo condições plenas na idade adulta. ${ }^{8}$

Para desenvolver um modelo assistencial que se caracterize pela excelência técnica, aliada aos valores de uma relação clínica eticamente adequada e que respeite a crescente autonomia dos adolescentes, é importante visualizar a prestação de serviços destinados aos jovens através de sua perspectiva e buscar estratégias 
que melhor satisfaçam suas necessidades. Com este objetivo, o presente estudo procura identificar o conhecimento, por parte dos adolescentes, dos limites éticos à confidencialidade e, em quais situações clínicas os participantes admitem a quebra de confidencialidade das informações coletadas na consulta médica.

\section{Métodos}

Com um delineamento transversal, contemporâneo, qualiquantitativo e exploratório-descritivo, o estudo foi realizado nos meses de novembro e dezembro de 2000, pesquisando as opiniões e comportamentos de 7 II universitários, por meio de um questionário anônimo, elaborado especificamente para a investigação, contendo perguntas abertas e fechadas sobre a confidencialidade na assistência à saúde?

Considerando que a Society for Adolescent Medicine preconiza que a assistência a este grupo etário deve direcionar-se a pessoas cuja idade vai dos 10 aos 25 anos $^{10}$ e que o Código Civil brasileiro vigente na época do estudo" fixava a maioridade civil aos 21 anos (fato que muda as exigências legais em relação à autonomia), optamos por pesquisar universitários até este limite de idade. Desta forma, a população em estudo constituiu-se de adolescentes entre 16 e 21 anos completos, cursando o $2^{\circ}$ semestre das Faculdades de Administração de Empresas, Direito, Enfermagem, Medicina e Odontologia da Pontifícia Universidade Católica do Rio Grande do Sul (PUCRS).

O fator em estudo foi o conhecimento dos universitários sobre os limites éticos da confidencialidade das informações em saúde. 0 desfecho avaliado foi a admissão da quebra da confidencialidade em situações clínicas hipotéticas, comuns na morbidade dos adolescentes, tais como: vida sexual ativa, gravidez não planejada, doenças sexualmente transmissíveis, homossexualidade, violência física e abuso sexual, anorexia nervosa e bulimia, risco de suicídio e uso de drogas.

O banco de dados foi construído com a utilização do programa Epi-Info, versão 6.04 (CDC/OMS, 1996) e foi analisado através dos recursos estatísticos do Epi-Info 6.04 e do Microsoft ${ }^{\hat{a}}$ Excel, 1997.

Para a descrição da população estudada, utilizaram-se os seguintes tratamentos estatíisticos: a) para as variáveis categóricas: tabelas de freqüência e proporções; b) para as variáveis numéricas: média e desvio-padrão; c) para avaliação de associações entre variáveis categóricas: o teste do Qui-quadrado e o teste exato de Fischer; d) para comparações entre médias e entre os grupos foram utilizados: o teste $t$ de Student para comparações entre dois grupos, cujas variáveis apresentaram distribuição simétrica e análise de variância (ANOVA) para comparações entre mais de dois grupos. Nas tabelas estão apresentados apenas os resultados descritivos; as diferenças e associações encontradas estão relatadas ao longo do texto, quando pertinentes.

O projeto de pesquisa foi aprovado pelo Comitê de Ética em Pesquisa da PUCRS; registro n 00/805, em 31 de outubro de 2000.

\section{Resultados}

\section{Características da população estudada}

De acordo com dados oficiais da PUCRS, matricularam-se nos cursos pesquisados, no primeiro semestre de 2000, 824 alunos com até 21 anos de idade. $O$ número de alunos efetivamente pesquisados alcançou $86,3 \%(n=7 \mid \mathrm{I})$ da população em estudo; sendo que $48,1 \%$ (342) pertencem ao sexo masculino e $51,9 \%$ (369) ao sexo feminino. A taxa de absenteísmo em sala de aula foi de $11 \%(91)$ e as negativas em participar da pesquisa representaram $2,7 \%(22)$.

A média etária geral foi de $18,84 \pm 1,13$ ano e a média para cada sexo, situou-se em 18,92 anos para o sexo masculino e em 18,77 anos para o feminino $(p>0,05)$. A mediana ficou em 19 anos de idade. Quanto ao estado civil, 98,2\% (698) são solteiros: 47,8\% do sexo masculino e $52,2 \%$ do sexo feminino, enquanto ।,8\% (9 homens e 4 mulheres) são casados.

Do total de 7II adolescentes, 175 (24,6\%) cursam Administração de Empresas, 392 (55,l\%) fazem Direito, 31 (4,4\%) pertencem à Enfermagem, 61 (8,6\%) à Medicina e 52 (7,3\%) à Odontologia. Alunos do sexo feminino predominam nas faculdades de Enfermagem (93,6\%), Odontologia (70,6\%), Medicina $(65,6 \%)$ e Direito $(51,0 \%)$, enquanto na Administração de Empresas encontra-se predomínio de alunos do sexo masculino $(63,5 \%)$

Moram com os pais 554 estudantes (78\%), dos quais, 267 $(48,2 \%)$ são do sexo masculino e $287(51,8 \%)$ do sexo feminino. Vivem com o cônjuge $1,5 \%$ dos alunos, sete homens e quatro mulheres, enquanto cinco estudantes $(0,7 \%)$ moram com um parceiro estável. Os restantes moram sozinhos (7,3\%), com colegas ou amigos (4,4\%) ou com outros familiares (8\%) como irmãos, tios, primos e avós.

Enquanto a maior parte dos estudantes de Enfermagem (93,5\% - 29), Administração de Empresas (82,3\% - 144) e Direito (80,6\% - 315) moram com os pais, na Medicina e Odontologia estas porcentagens baixam, respectivamente, para 59\% (36) e 57,7\% (30). Esta diferença mostrou-se estatisticamente significativa ( $p<$ $0,001)$.

Declararam a renda familiar $353(49,6 \%)$ universitários, a qual variou de $R \$ 700,00$ a $R \$ 50.000,00$, com média de $R \$ 5.734,47$ e mediana de $R \$ 4.000,00$.

Com referência a fontes de renda pessoal, 46 I (64,8\%) recebem dinheiro dos pais em forma de mesada ou conforme a necessidade, 188 (26,4\%) têm emprego, 32 (4,5\%) recebem pensão, 18 (2,5\%) fazem estágio remunerado ou monitoria e 12 $(1,8 \%)$ declararam ter outras fontes de renda, como rendimentos de aplicações financeiras, patrocínio esportivo, vendas, exército e músico profissional.

Dos 188 (26,4\%) universitários que possuem emprego, 95 (54,3\%) são alunos de Administração de Empresas e 89 (22,7\%) são alunos de Direito. Nos cursos da área da saúde, somente um acadêmico de Medicina (I,6\%), um de Odontologia (1,9\%) e dois alunos de Enfermagem (6,5\%) têm emprego, diferença que se mostrou estatisticamente significativa $(p<0,001)$. 
LOCH JA Et AL.

\section{Assistência à saúde}

Mais da metade (51,3\% - 365) dos estudantes vai periodicamente ao médico para supervisão de saúde, mesmo não estando enfermos. Destes, 36,5\% (125) são homens e 65\% (240) são mulheres. Mais alunos de Medicina (68,9\%) e de Enfermagem $(64,5 \%)$ fazem consultas preventivas do que alunos de Direito (48,7\%), Administração de Empresas (49,1\%) e Odontologia $(51,0 \%)$, diferença estatisticamente significativa $(p<0,026)$.

Nos últimos dois anos, 633 alunos (89,3\%) necessitaram consultar pelo menos um médico por um problema de saúde, representando 84,8\% (289) dos homens e 93,5\% (344) das mulheres. Duzentos e dezenove alunos do sexo masculino $(70,4 \%)$ e 278 do sexo feminino $(78,5 \%)$ declararam haver consultado o mesmo profissional anteriormente, num total de 497 respostas (74,7\%), enquanto que 168 universitários (25,3\%) não haviam consultado o mesmo médico e 46 (6,5\%) não responderam a esta pergunta.

A Tabela | apresenta as freqüências dos médicos consultados em relação ao sexo dos alunos pesquisados.

\begin{tabular}{|c|c|c|c|c|c|c|}
\hline \multicolumn{7}{|c|}{$\begin{array}{l}\text { Tabela I - Freqüência dos médicos consultados de acordo com o } \\
\text { sexo dos alunos pesquisados }\end{array}$} \\
\hline \multirow[t]{3}{*}{ Médicos consultados } & \multicolumn{4}{|c|}{ Alunos } & & \\
\hline & \multicolumn{2}{|c|}{ Sexomasculino } & \multicolumn{2}{|c|}{ Sexofeminino } & \multicolumn{2}{|c|}{ Total } \\
\hline & $\mathbf{N}$ & $\%$ & $\mathbf{N}$ & $\%$ & $\mathrm{~N}$ & $\%$ \\
\hline $\begin{array}{l}\text { Clínico Geral } \\
\text { Dermatologista } \\
\text { Ginecologista } \\
\text { Otorrinolaringologista } \\
\text { Oftalmologista } \\
\text { Pediatra } \\
\text { Traumato-ortopedista } \\
\text { Urologista } \\
\text { Outros* }\end{array}$ & $\begin{array}{c}139 \\
10 \mid \\
- \\
35 \\
23 \\
27 \\
42 \\
17 \\
53\end{array}$ & $\begin{array}{c}40,8 \\
29,6 \\
- \\
10,3 \\
6,7 \\
7,9 \\
12,3 \\
5,0 \\
15,5\end{array}$ & $\begin{array}{l}124 \\
151 \\
222 \\
28 \\
33 \\
29 \\
13 \\
13 \\
79\end{array}$ & $\begin{array}{r}33,6 \\
40,9 \\
60,2 \\
7,6 \\
9,0 \\
7,9 \\
3,5 \\
3,5 \\
21,4\end{array}$ & $\begin{array}{r}263 \\
252 \\
222 \\
63 \\
56 \\
56 \\
55 \\
30 \\
132\end{array}$ & $\begin{array}{l}37,0 \\
35,4 \\
31,2 \\
8,9 \\
7,9 \\
7,9 \\
7,7 \\
4,2 \\
18,6\end{array}$ \\
\hline
\end{tabular}

*Outros: Pneumologista, Endocrinologista, Neurologista, Gastrenterologista, Psiquiatra, Cardiologista, Nefrologista, Cirurgiäo

Geral e Plástico, Homeopata, Proctologista, Alergista, Nutrólogo, Hematologista, Fisiatra, Oncologista e Reumatologista.

\section{Limites à confidencialidade}

Com o objetivo de verificar o conhecimento por parte dos adolescentes dos limites à confidencialidade, perguntou-se quais as informações prestadas em uma consulta que, no entender deles, poderiam ou deveriam ser reveladas pelo médico para a família, parceiros ou amigos, como mostra a Tabela 2.

\section{Limites à confidencialidade em situações clínicas específicas}

Quando colocados como protagonistas de situações hipotéticas e perguntados em quais delas o médico poderia ou deveria revelar os fatos à família, parceiros ou amigos, mesmo sem o seu consentimento, os universitários responderam conforme o demonstrado na Tabela 3.

\section{Discussão}

A participação de adolescentes como sujeitos de pesquisa proporciona informações relevantes para diversas áreas da assistência, permitindo, de maneira apropriada e realística, atender tanto às necessidades de saúde do grupo como ao desenvolvimento de políticas e programas de prevenção e tratamento ${ }^{12}$.

Garantir a confidencialidade das informações é muito importante na pesquisa com adolescentes, porque suas causas de adoecimento relacionam-se a questões sensíveis, como os comportamentos de risco e a sexualidade. Uma vez que o presente estudo inclui perguntas sobre estes assuntos, procuramos utilizar estratégias eticamente adequadas para abordá-los ${ }^{12-14}$. Para garantir o anonimato dos dados coletados, optamos pela obtenção de um consentimento informado de forma verbal, que foi submetido e aprovado pelo Comitê de Ética em Pesquisa. Metodologicamente, o consentimento verbal ajudou para diminuir um viés potencial do estudo, pois os adolescentes, quando passíveis de identificação, respondem com menor veracidade a perguntas sobre questões íntimas ${ }^{13,15}$.

Avaliamos as vantagens e as limitações do ambiente universitário como cenário da pesquisa. Consideramos vantajoso o delineamento do trabalho, que permite delimitar uma população para o estudo (ingressantes na PUCRS no $1^{\circ}$ semestre de 2000, em cursos estabelecidos) e estudá-la na totalidade, além de que a

Tabela 2 - Informaçóes que o médico pode ou deve revelar à família, parceiros(as) ou amigos ( $\mathrm{n}=7 \mathrm{II}$ )

\begin{tabular}{|c|c|c|c|c|c|c|}
\hline \multirow[t]{2}{*}{ Que informações você acha que seu médico pode ou deve revelar? } & \multicolumn{2}{|c|}{ Para sua Família } & \multicolumn{2}{|c|}{ Parceiro/a ou namorado/a } & \multicolumn{2}{|c|}{ Amigos ou conhecidos } \\
\hline & $\mathrm{n}$ & $\%$ & $n$ & $\%$ & $\mathrm{n}$ & $\%$ \\
\hline Todas as informaçōes que você deu na consulta & 143 & 20,1 & 28 & 3,9 & 4 & 0,6 \\
\hline $\begin{array}{l}\text { Qualquer informação que ele, médico, julgar que eles devem saber, } \\
\text { mesmo que vocênão autorize }\end{array}$ & 258 & 36,3 & 60 & 8,4 & $\|$ & 1,5 \\
\hline Só as informações que você consentir que sejam divulgadas & 587 & 82,6 & 319 & 44,9 & 203 & 28,6 \\
\hline $\begin{array}{l}\text { Só as informações que constituem risco para sua vida } \\
\text { (câncer, Aids, doenças mentais) }\end{array}$ & 509 & 71,6 & 218 & 30,7 & 41 & 5,8 \\
\hline $\begin{array}{l}\text { Informações que constituem risco para a vida de outras pessoas conhecidas } \\
\text { (doenças contagiosas, Aids, intenção de homićídio) }\end{array}$ & 514 & 72,3 & 303 & 42,6 & 114 & 16 \\
\hline
\end{tabular}




\begin{tabular}{|c|c|c|}
\hline \multirow[t]{2}{*}{ Situação Hipotética } & \multicolumn{2}{|c|}{ Omédico poderia ou deveria revelar } \\
\hline & $\mathbf{n}$ & $\%$ \\
\hline "Tenho idéias de cometer suicídio" & 601 & 85,0 \\
\hline "Estou sendo vítima de violência física" & 593 & 84,2 \\
\hline "Estou com bulimia ou anorexia nervosa" & 573 & 81,3 \\
\hline "Estou sendo vítima de abuso sexual" & 576 & 81,7 \\
\hline "Sou HIV positivo(a)" ou "Tenho Aids" & 408 & 57,9 \\
\hline "Sou usuário(a) de álcool ou drogas" & 366 & 51,7 \\
\hline "Tenho uma doença sexualmente transmitida" & 315 & 44,7 \\
\hline "Estou grávida"/"Engravidei minha namorada" & 237 & 33,6 \\
\hline "Sou homossexual"" & 146 & 20,7 \\
\hline "Estou tendo relações sexuais" & 110 & 15,6 \\
\hline
\end{tabular}

variedade demográfica que uma escola propicia, ajuda o entendimento normativo das questões de saúde dos adolescentes. ${ }^{13}$ Como desvantagem, salientamos que este tipo de cenário exclui os adolescentes de maior risco: aqueles que estão fora do ambiente educacional $^{16}$. Da mesma forma, outras faixas etárias, incluindo a adolescência inicial e média, também poderiam apresentar resultados diferentes dos encontrados nesta pesquisa.

Os estudantes pesquisados são oriundos de um estrato social, cultural e economicamente privilegiado, considerando a renda familiar declarada e as estatísticas que revelam que apenas 20\% da população brasileira possui 1 | anos ou mais de escolarização ${ }^{17}$. Portanto, o estudo envolveu uma população diferenciada quanto às características gerais dos adolescentes brasileiros e sugerimos que os resultados não sejam generalizados para a totalidade da adolescência no país. Salientamos, assim como Feijó e Chaves, que esta limitação metodológica é inerente aos estudos sobre o comportamento humano, já que características culturais, hábitos regionais e de grupos sociais definem comportamentos distintos também entre os adolescentes ${ }^{18}$. Assim sendo, preocupamo-nos em garantir validade interna ao trabalho, aliando à objetividade do método quantitativo a análise qualitativa das opiniões dos respondentes, mantendo sua perspectiva quando da descrição dos possíveis motivos para os resultados encontrados. Se considerarmos a afirmação contida na Carta de Jacarta/OMS", de que "O acesso à instrução e à informação é essencial para alcançar a participação eficaz e o direito de voz das pessoas e das comunidades", os jovens estudados preenchem as condições de servirem como portavozes de seus pares quando o objetivo é pesquisar um modelo de assistência atento às necessidades de individuação e privacidade dos adolescentes como grupo.

\section{Assistência à saúde}

Apenas $51,3 \%$ dos respondentes costumam fazer revisão de saúde, sugerindo que, mesmo numa camada populacional, econômica e socialmente privilegiada como esta, somente metade dos adolescentes está tendo atitudes preventivas. Ressaltamos que, entre aqueles que vão periodicamente ao médico, há uma proporção de 1,85 mulheres para cada homem. Diferentemente de nosso achado, dados da literatura mostram que a porcentagem de adolescentes norte-americanos que vai periodicamente ao médico chega a $80 \%$, na mesma faixa etária ${ }^{20}$. Esta diferença significativa pode estar relacionada a questões culturais de entendimento da importância da prevenção no contexto saúde-doença e condicionada por dificuldades de acesso ou por falta de renda própria por parte dos adolescentes deste estudo.

Mesmo sem o hábito de fazer prevenção, 89,3\% dos jovens necessitaram consultar pelo menos um médico nos últimos dois anos. $O$ profissional mais procurado pelas mulheres foi o ginecologista $(60,2 \%)$ e, pelos homens, o clínico geral $(40,8 \%)$. O pediatra foi consultado por 7,9\% dos rapazes e 7,9\% das moças. Outros especialistas, espontaneamente mencionados, estão relacionados com problemas freqüentes na adolescência, como afecções dermatológicas e traumatismos decorrentes da prática esportiva, além de problemas de visão e audição, como foi mostrado na Tabelal.

Um total de $74,7 \%$ dos respondentes já havia consultado estes profissionais anteriormente, configurando um vínculo de relacionamento positivo, fator importante para facilitar o acesso e a continuidade da assistência ao adolescente como atestam vários traba||$^{\prime 1,21}$. Na pesquisa de Ginsburg et al., os adolescentes referiram, da mesma maneira que os participantes deste estudo, que estando satisfeitos com seus cuidadores, buscam mais assistência e seguem com mais fidelidade as orientações de prevenção e tratamento. ${ }^{21}$

\section{Limites à confidencialidade}

Para verificar quais limites à confidencialidade os adolescentes reconheciam como válidos, perguntou-se quais informações prestadas em uma consulta poderiam ou deveriam ser reveladas pelo médico para a família, parceiros ou amigos. Para a formulação da pergunta, foram utilizados os critérios éticos para a quebra de confidencialidade propostos por Schiedermayer, em 199122. O autor utiliza os princípios bioéticos de Beauchamp e Childress ${ }^{23}$ para fundamentar eticamente a quebra de sigilo, afirmando que ela somente pode ser admitida em quatro circunstâncias gerais: a) quando houver grande probabilidade de um sério dano físico a uma terceira pessoa identificável e específica, estando justificada pelo princípio da Não-Maleficência; b) quando um benefício real para o paciente resultar desta revelação, baseando-se esta decisão no princípio de Beneficência; c) quando for o último recurso, depois de esgotadas todas as abordagens para respeitar a Autonomia; e d) quando a mesma decisão de revelação possa ser utilizada em outras situações com características idênticas, independentemente da posição social do paciente, contemplando o princípio de Justiça22.

Como pode ser visto na Tabela 2, os universitários reconhecem a situação eticamente adequada de necessidade de consentimento e autorização do paciente para a revelação das informações compartilhadas na consulta, tanto que $82,6 \%$ consideram lícito que o médico revele informações à família; $44,9 \%$ e $28,6 \%$, respectivamente, que revele aos parceiros e aos amigos, desde que haja a permissão do paciente. Consistente com esta posição, apenas 20,1\% acham que o médico tem o direito de revelar à família todas 
LOCH JA ET AL.

as informações dadas em consulta, porcentagem que baixa para $3,9 \%$ e $0,6 \%$, quando se trata de contar a parceiros ou amigos.

Os alunos também identificam os limites éticos à confidencialidade nas situações de risco à vida. Quando há riscos à sua integridade pessoal $(71,6 \%)$ ou à de terceiros $(72,3 \%)$, cerca de três quartos dos respondentes admitem a revelação não autorizada para a família. A revelação aos parceiros foi considerada correta por 30,7\%, nos riscos à própria vida e por $42,6 \%$, no risco a terceiros. Aos amigos, apenas $5,8 \%$ dos respondentes concordam que o médico poderia revelar informações não autorizadas quando há ameaça à integridade do paciente e 16,0\% concordam quando há riscos para outrem.

É interessante assinalar que um terço $(33,6 \%)$ dos universitários confere ao médico a autoridade para revelar, sem consentimento, aqueles fatos (mesmo não representando sério risco à vida) que ele, médico, julgue importantes que a família tome conhecimento. Este achado corrobora com a idéia de que o pressuposto básico para a confidencialidade é a confiança24. Vários autores defendem que as informaçõos sensíveis compartilhadas entre médico e paciente são consideradas "segredos de confiança"25-26. Nestas situações, a informação tem um caráter instrumental, servindo a um objetivo específico e a única justificativa para a quebra do sigilo é melhor alcançar este propósito. Dito de outra forma, o adolescente revela informações sensíveis por necessidade de ajuda ou para tentar resolver um problema e, assim sendo, confia que o médico, ao inteirar-se da questão, possa ajudá-lo de fato e, se para isto houver necessidade de revelar à família, ele espera que o médico o faça.

\section{Limites à confidencialidade em situações clínicas específicas}

Partindo da generalização sobre os limites à confidencialidade acima discutida, o questionário propiciou o estudo de situações específicas relacionadas aos comportamentos de risco, visando identificar mais precisamente em que casos a autoridade conferida ao profissional para revelar informações não autorizadas teria maior consistência.

Nos casos de ideação suicida, violência física, anorexia nervosa, bulimia e abuso sexual, cerca de $80 \%$ dos respondentes mostraram-se dispostos a aceitar a quebra da confidencialidade, mesmo sem prévio consentimento, bem como no caso de HIV/Aids (57,9\%), reconhecendo os riscos potenciais à sua integridade e a necessidade de ajuda para lidar com estas questões. Para aquelas situações que envolvem dependência química (uso de drogas), $51,7 \%$ dos adolescentes aceitam as revelações não autorizadas, ficando divididos entre o direito à confidencialidade e a necessidade de ajuda familiar. Já nas questões relacionadas à sexualidade (gravidez, homossexualidade e vida sexual ativa), as taxas de concordância com a quebra da confidencialidade caem abaixo de $30 \%$, para os sexos combinados (Tabela3).

Quando estes resultados foram analisados em relação ao gênero dos respondentes, somente nas questões relativas à sexualidade foram encontradas associações estatisticamente significati- vas $(p<0,000 I)$, utilizando-se o teste do Qui-quadrado. As mulheres demandam maior grau de privacidade que os homens nas situações pesquisadas. Enquanto 52,4\% dos alunos do sexo masculino admitiriam a quebra não autorizada do sigilo no caso de serem portadores de DST, apenas $37,6 \%$ do sexo feminino o fariam. Em situação de gravidez, $43 \%$ dos homens e $25 \%$ das mulheres acham que o médico pode revelar; na homossexualidade, $31,5 \%$ dos homens e 10,9\% das mulheres, e no caso de vida sexual ativa, $24,5 \%$ dos alunos contra somente $7,3 \%$ das alunas concordariam com a revelação não autorizada. Este achado pode ser explicado com base no conceito de tipificação ou estereótipo de papel sexual ${ }^{27}$. As participantes do estudo se autoconcedem um conjunto de qualidades que definem a condição de ser mulher no seu contexto cultural, reconhecendo na privacidade e no recato características inerentes deste papel, estando conscientes da repercussão negativa para o sexo feminino de assumir sua sexualidade fora dos padrões sociais vigentes.

Apesar desta diferença de gênero, homens e mulheres do estudo consideram as questões de sexualidade como as mais pessoais e sensíveis, demandando um grau de sigilo maior por parte dos profissionais de saúde.

A privacidade e confidencialidade da assistência ao adolescente tem sido avaliada e discutida mais profundamente em países onde o individualismo e os direitos pessoais são proeminentes no sistema normativo jurídico. Nos Estados Unidos, por exemplo, associações médicas comprometidas com a assistência ao adolescente, como a American Medical Association, a American Academy of Pediatrics, a American Public Health Association, o American College of Obstetricians and Gynecologists e a Society for Adolescent Medicine dizem que "há um consenso de que os adolescentes devem ter acesso a serviços de assistência que sejam confidenciais e que o envolvimento, consentimento ou notificação aos pais não deveria ser uma barreira para este atendimento"28.

No Brasil, o Código de Ética Médica (art. 103) veda ao médico "revelar segredo profissional referente a paciente menor de idade, inclusive a seus pais ou responsáveis legais, desde que o menor tenha capacidade de avaliar seu problema e de conduzir-se por seus próprios meios para solucioná-lo, salvo quando a não-revelação possa acarretar danos ao paciente"29. A Sociedade Brasileira de Pediatria, em documento de 1999, ratifica este posicionamento e enfatiza o direito do adolescente à confidencialidade, declarando que "os pais ou responsáveis somente serão informados sobre o conteúdo das consultas como, por exemplo, nas questões relacionadas com a sexualidade e prescrição de métodos contraceptivos, com o expresso consentimento do adolescente." ${ }^{30 .}$

Todas estas entidades reconhecem que o envolvimento da família nas questões de saúde do adolescente é altamente desejável e deve ser incentivado, porém os limites desta participação devem ficar claros para a família, para o jovem e para o profissional que os assiste. Este estudo mostra que os adolescentes pesquisados têm consciência da necessidade da participação familiar nas questões mais graves e aponta claramente sua intenção de fazê-lo nas situações em que eles acreditam que isto seja necessário. Portanto, 
os resultados evidenciam que os adolescentes estudados utilizam um critério de ponderação entre seus direitos e o risco associado a determinadas situações de morbidade, assumindo a possibilidade de redução do direito à privacidade toda vez que um valor maior esteja ameaçado.

\section{ConclusÃo}

Podemos concluir, de acordo com os objetivos pretendidos, que os adolescentes do estudo estabelecem graus diferentes sobre o valor da confidencialidade na assistência a sua saúde. A exigência, por parte do adolescente, de maior ou menor privacidade depende essencialmente do motivo para o atendimento médico. Os universitários reconhecem e aceitam que as informações obtidas nas consultas possam ser comunicadas a terceiros quando o próprio paciente dê autorização para a revelação. O fato de o médico poder dividir a informação não autorizada com outras pessoas é também considerado factível, sendo que os participantes admitem mais facilmente a quebra da confidencialidade nas situações que envolvem riscos para a própria vida e para a vida de terceiros. Sua resposta não é uniforme em casos de dependência química e dificilmente aceitam a revelação não autorizada nos aspectos que se referem a sua sexualidade.

\section{Agradecimentos}

Os autores agradecem à Dra. Lúcia Pellanda pelo auxílio, sugestões e competência na análise estatística dos dados.

\section{Conflito de interesse: não há}

\section{SUMMARY}

Privacy and CONFidentiality In Adolescent health CaRe: PERCEPTIONS AND BEHAVIOR OF A GROUP OF 7 I I COLLEGE STUDENTS

OBJECTIVE. To understand the opinion and behavior of a group of college students about the degree of privacy considered appropriate in several clinical settings and in which situations breach of confidentiality is admitted.

METHODS. An anonymous questionnaire about confidentiality in clinical settings was answered by $7 / 1$ college students. The study had a transversal, descriptive, qualitative-quantitative design. We studied recognition of the ethical criteria for confidentiality limitation, and in which situations, common in adolescent morbidity, disclosure of this information is accepted. Data analysis used EpiInfo 6.04 and Microsoft Excel, 1997. The research was approved by PUCRS' IRB.

RESULTS. The ideal situation for disclosing information was considered by $82 \%$ of the adolescents as the previous granting of authorization, which differs from other forms of breaching confidentiality, In cases of non-authorized disclosure, most of them admitted that it in case of suicidal ideation (85\%), violence (84.2\%), sexual abuse (81.7\%), nervous anorexia (81.3\%) and risk of life to third parties (72.3\%). Half of them agree to it in HIV/AIDS (57.9\%), drug abuse (51.7\%) and STD (44.7\%) situations; less than one third accept it in situations of pregnancy (33.6\%), homosexuality (20.7\%) and sexual activity (15.6\%).

Conclusion. Participants assigned different grades to the value of confidentiality in their health care, accepting that information may be disclosed to others when the patient authorizes it. The higher the risk to their integrity, the easier it was for them to admit nonauthorized disclosure of information, however in aspects related to their sexuality disclosure is practically denied. [Rev Assoc Med Bras 2007; 53(3): 240-6]

KEY WORDS: Adolescent behavior. Privacy. Confidentiality.

\section{REFERÊNCIAS}

I. English A, Kapphahn C, Perkins J, Wibbelsman C). Meeting the health care needs of adolescents in managed care: a background paper. Adolesc Health. 1998;22:278-92.

2. Klein JD, Slap GB, Elster AB, Schonberg SK. Access to health care for adolescents: a position paper of the Society for Adolescent Medicine. Adolesc Health. 1992;13:162-70.

3. American Medical Association. Confidential health services for adolescents. Council on Scientific Affairs, American Medical Association. JAMA. 1993:269:|420-4.

4. Felice ME, Feinstein RA, Fisher M, Kaplan DW, Olmedo LF, Rome ES. [American Academy of Pediatrics. Committee on Adolescence]. Contraception in adolescents. Pediatrics. 1999:104(5 Pt 1):||6|-6.

5. Loch JA. Confidencialidade: natureza, características e limitações no contexto da relação clínica. Bioética. 2003;1 1:1:51-64.

6. Francisconi CF, Goldim JR. Aspectos bioéticos da confidencialidade e privacidade. In: Costa SIF, Oselka G, Garrafa V, coordenadores. Iniciação à bioética. Brasília: Conselho Federal de Medicina; 1998. p. 269-84.

7. Winslade W]. Confidentiality. In: Reitch WT, editor. Encyclopedia of bioethics. New York: Simon \& Schuster Macmillan; 1995. p.45I-59.

8. Clotet J, Loch JA. Capacidade para tomar decisões sanitárias: autonomia moral versus autonomia legal. In: Urban CA, coordenador. Bioética clínica. Rio de Janeiro: Revinter; 2003. p.44-7.

9. Loch JA. La confidencialidad en la asistencia a la salud del adolescente: percepciones y comportamientos de un grupo de universitarios de Porto Alegre, RS Brasil [dissertação] Santiago, Chile: Universidad de Chile/Organización Panamericana de la Salud; 200I.

10. Society for Adolescent Medicine. Position statement on HIV and youth. J Adolesc Health. 1995; 16:413.

11. Brasil. Código Civil. Lei n. 3.071, de 01.01.1916, atualizada e acompanhada de legislação complementar. $7^{\text {a }}$ ed. São Paulo: Saraiva; 2001

12. English A. Guidelines for adolescent health research: legal perspectives. J Adolesc Health. 1995; 17:277-86.

13. Gans JE, Brindis CD. Choice of research setting in understanding adolescent health problems. J Adolesc Health. 1995; 17:306-13.

14. Brasil. Ministério da Saúde. Conselho Nacional de Saúde. Resolução 196/96. Diretrizes e Normas Regulamentadoras de Pesquisas envolvendo Seres Humanos. Brasília: O Conselho; 1996.

15. Siegel DM, Aten MJ, Roghmann KJ. Self-reported honesty among middle and high school students responding to a sexual behavior questionnaire. J Adolesc Health. 1998;23:20-8.

16. Maddaleno M. La salud de los adolescentes y jóvenes. In: Benguigui Y, Land S, Paganini JM, Yunes J. Acciones de salud maternoinfantil a nivel local: según las metas de la cumbre mundial en favor de la infancia. Washington: Organización Panamericana de la Salud; 1999. p.185217.

17. Instituto Brasileiro de Geografia e Estatística. Pesquisa Nacional por Amostra de Domicílios. Educação. Pessoas com 10 anos ou mais de idade, total e por sexo, segundo os grupos de estudo 1999. [citado 21 
LOCH JA ET AL.

Fev 200I]. Disponível em:URL:http://www.lbge.gov.br/ibge/ estatistica/populacao/trabalhoerendimento/pnad99/sintese/images/ tabelaegrafico3_2b.shtm.

18. Feijó RB, Chaves MLF. Comportamento suicida. In: Costa COM, Souza RP, organizadores. Adolescência: aspectos clínicos e psicossociais. Porto Alegre: Artmed; 2002. p.398-408

19. Brasil. Ministério da Saúde. Referências para leitura. Promoção da Saúde 1999;1:35-46.

20. Klein JD, McNulty M, Flatau CN. Adolescents' access to care: teenagers' self-reported use of services and perceived access to confidential care. Arch Pediatr Adolesc Med. 1998;152:676-82.

21. Ginsburg KR, Menapace AS, Slap GB. Factors affecting the decision to seek health care: the voice of adolescents. Pediatrics. 1997;100:922 30

22. Schiedermayer DL. Guarding secrets and keeping counsel in computer age. I Clin Ethics. 1991:2:33-4.

23. Beauchamp TL, Childress JF. Principles of biomedical ethics. $4^{\text {th }}$.ed. New York: Oxford University; 1994.

24. Styffe EJ. Privacy, confidentiality, and security in clinical information systems: dilemmas and opportunities for the nurse executive. Nurs Adm Q. 1997:21:21-8.
25. Kottow MH. Introducción a la bioética. Santiago: Editorial Universitaria; 1995.

26. Luban D. Secrecy and confidentiality. In: Becker LC, Becker CB, editors. Encyclopedia of ethics. New York: Garland; 1992. p.II31-3.

27. Bee H. A criança em desenvolvimento. $7^{\text {a }}$ ed. Porto Alegre: Artes Médicas; 1996. p.298-230.

28. American Academy of Pediatrics. The adolescent's right to confidential care when considering abortion. [American Academy of Pediatrics. Committee on Adolescence]. Pediatrics; 1996;97:746-5।

29. Brasil. Conselho Federal de Medicina. Resolução CFM n |.246/88. Código de ética médica. Rio de Janeiro: O Conselho; 1988.

30. Oselka GW, Troster El. Aspectos éticos do atendimento do adolescente. Rev Assoc Med Bras. 2000:46:306-7.

Artigo recebido: 13/08/06

Aceito para publicação: 08/02/07 